\title{
De la géopolitique à l'humanitaire : le cas du Kurdistan d'Irak
}

\section{Hamit Bozarslan}

\section{(2) OpenEdition}

1 Journals

\section{Édition électronique}

URL : http://journals.openedition.org/conflits/425

DOI : $10.4000 /$ conflits.425

ISSN : $1777-5345$

Éditeur :

CCLS - Centre d'études sur les conflits lilberté et sécurité, L'Harmattan

Édition imprimée

Date de publication : 15 octobre 1993

ISSN : 1157-996X

Référence électronique

Hamit Bozarslan, «De la géopolitique à l'humanitaire : le cas du Kurdistan d'Irak », Cultures \& Conflits [En ligne], 11 | automne 1993, mis en ligne le 13 mars 2006, consulté le 30 mars 2021. URL : http:// journals.openedition.org/conflits/425 ; DOI : https://doi.org/10.4000/conflits.425

Ce document a été généré automatiquement le 30 mars 2021.

Creative Commons License 


\title{
De la géopolitique à l'humanitaire : le cas du Kurdistan d'Irak
}

\author{
Hamit Bozarslan
}

La Guerre du Golfe a pris les observateurs au dépourvu ${ }^{1}$ : non seulement elle a déplacé l'axe de la politique internationale, dans un monde largement "post-communiste", en montrant qu'une guerre pouvait avoir lieu, non pas entre les deux "blocs" comme on l'avait craint durant des décennies, mais bien entre le Nord et un pays du Sud, longtemps soutenu et armé par l'Occident pour faire face à un Iran diabolisé, avant d'être à son tour diabolisé ; mais, elle s'est arrêtée à un moment où elle était presque parvenue à l'un de ses objectifs à peine voilés : la destruction de la garde républicaine et le renversement d'un régime, qualifié "d'hitlérien". Au contraire, le cessez-le-feu signé entre le Conseil de Sécurité de l'ONU et Bagdad a laissé à ce dernier toute latitude pour écraser les révoltes kurde au nord et chi'ite au sud qui ont éclaté au lendemain de la guerre ${ }^{2}$. Un nouveau revirement a succédé à cette "neutralité" par rapport aux "affaires internes" irakiennes: l'Occident, la Maison Blanche en tête, a réalisé une "opération humanitaire" ("Provide Comfort") d'une envergure inconnue jusque-là pour venir en aide aux Kurdes. L'objectif de cette opération était, au début, limité : arrêter l'exode qui avait suivi l'écrasement de leur révolte en mars 1991 et qui avait poussé la moitié de la population vers l'Iran ou la Turquie. L'expérience ainsi entamée a cependant très largement dépassé les intentions initiales des alliés occidentaux ainsi que celles des Kurdes eux-mêmes : elle a commencé par une résolution, officialisant, pour la première fois, le droit d'ingérence dans un pays membre de l'ONU pour des raisons humanitaires et par la mise en place d'une zone de protection pour les populations civiles pour aboutir de facto à la création d'une entité politique kurde qui a, depuis, enregistré à son actif des élections pluralistes, la constitution d'un gouvernement, la déclaration d'un Etat fédéral et l'amorce d'une réunification des forces militaires. Le gouvernement de la région kurde s'est approprié la plupart des prérogatives du Front du Kurdistan et du pouvoir central de Bagdad ${ }^{3}$. Sans aller jusqu'à reconnaître l'Etat fédéral, la Maison Blanche s'est voulue cependant gardienne de cet état de fait. Devant tant de volte-face, les observateurs se sont interrogés sur le cynisme, la sincérité ou encore les contradictions de la Maison Blanche, le protagoniste 
le plus important du conflit. Les deux premiers termes sont évidemment subjectifs et il n'est en effet pas exclu qu'ils aient tous deux déterminé les différentes phases de la politique américaine dans "l'affaire kurde". Faut-il pour autant chercher des contradictions dans l'attitude de l'administration américaine ou au contraire, y déceler une cohérence qui couvrirait plutôt, la période allant de la crise du Golfe à l'écrasement des révoltes kurde et chi'ite? D'autres questions émergent également: s'agissait-il d'une situation de fait, fruit de certains rapports de forces et d'une série de circonstances ou une première ébauche du nouveau paysage international après la Guerre Froide? La Maison Blanche inaugurait-elle, avec l'ingérence humanitaire, une nouvelle matrice dans les relations internationales pour en finir une fois pour tout avec la logique et la souveraineté indivisible et absolue des Etats ? Comment expliquer le fait que la Bosnie, pourtant reconnue internationalement, à la différence du Kurdistan, et admise à l'ONU, n'ait pu devenir un Kurdistan bis? Pourquoi le même scénario n'a-t-il pas été possible ailleurs dans le monde, au Cambodge ou en Somalie? Au lendemain de la Résolution $688^{4}$, Olivier Roy précisait qu'il ne pouvait y avoir de "diplomatie humanitaire" 5 . Il s'agissait en effet d'une diplomatie d'attente qui n'incluait nullement la volonté politique de résoudre le problème kurde. Au contraire, tout indique que les alliés n'ont, à aucun moment, considéré la question kurde comme l'une des questions politiques majeures du Moyen-Orient et qu'ils n'ont jamais eu l'intention de remettre en cause les frontières, y compris celles de l'Irak. La dimension, où le calcul politique ne gérait donc pas la logique humanitaire, même si, dans un deuxième temps, la volonté de maintenir la pression sur Saddam n'a guère manqué. Force est en effet de remarquer que "l'action humanitaire" s'inscrivait dans une logique de "day-to-day foreign policy", visant à gérer une situation devenue non maîtrisable, elle-même issue de la Guerre. C'est l'exode, largement spontané, provoqué par le spectre des armes chimiques, qui a forcé les capitales occidentales à agir. Mais, quelle que soit la volonté des secteurs "kurdophiles" des sphères politiques occidentales, deux questions n'en continuaient pas moins à déterminer l'extrême limite de l'attitude américaine et européenne et ce, même après la mise en place de l'action humanitaire : comment éviter la disparition de l'autorité centrale, non pas pour maintenir le régime de Saddam, mais pour prévenir une "libanisation" ou une nouvelle révolution "islamique" dans la région ? Compte tenu de la nature transfrontalière de la question et des revendications ultimes du nationalisme kurde, à savoir, la création d'un Etat englobant aussi les régions kurdes de la Syrie, de la Turquie et de l'Iran, comment contenir "l'humanitaire" dans un cadre strictement "non-irrédentiste", autrement dit, dans les limites étatiques de l'Irak ? Les aspects d'une intervention humanitaire L'action avait donc des objectifs restreints. Assistait-on, cependant, à la mise en oeuvre par des ONG, des Etats occidentaux ou ceux de la région, d'une mesure purement humanitaire, dépourvue de tout intérêt organisationnel, géopolitique ou encore hégémonique? Quels que soient les acteurs présents dans le processus de decision making, il serait singulièrement naïf de croire que les facteurs "extra-humanitaires" n'aient pas joué un rôle dans ce qu'il convient bel et bien de définir comme une innovation sur le droit international. Pour en saisir clairement l'importance, il convient d'analyser la période depuis la Guerre du Golfe comme un processus (qui se poursuit encore) sans s'arrêter uniquement aux premiers mois de l'expérience, les seuls, en fin de compte, qui soient marqués par "l'humanitaire". Vu sous cet angle, nous découvrirons de multiples enjeux, chacun lié à un type d'acteur. Il est évident que ces enjeux, nullement définis une fois pour toute, sont étroitement imbriqués et, de toute manière, limités par les intérêts et les moyens 
dont disposent les autres groupes impliqués. Sans aller jusqu'à souscrire aux accusations de "pompiers pyromanes" ou de "l'humanitaire, alibi de la realpolitik", nous remarquerons que certaines ONG se sont pleinement inscrites dans le jeu de pouvoir $^{6}$, sans pour autant remettre en cause la politique étrangère de leur pays qui avait permis à Saddam d'asseoir son pouvoir. Pour la première fois également les ONG ont pu considérablement amplifier leurs actions, multiplier le domaine de leurs actions, allant de la collecte de sommes importantes à la prise de décisions nationales et internationales. L'ensemble de l'opération a également montré que les ONG étaient devenues des entreprises rentables, alors même que le champ de l'action humanitaire, déjà marqué par des conflits, éclatait. Précisons aussi, indépendamment de l'adjectif par lequel on la définira (humanitaire, politique ou pragmatique), que la base de l'intervention n'était nullement juridique. Le droit international était (et reste) d'ailleurs mal outillé pour traiter d'une telle situation. Au contraire, il s'agissait, juridiquement parlant, d'une intervention dans les affaires "intérieures" d'un pays, Etat-membre de l'ONU qui, à la cessation des hostilités, devait normalement redevenir aussi souverain qu'un autre. L'accord signé par le Conseil conférait une légitimité officielle à l'action, mais ne la transformait nullement en une première juridique. Du côté arabe, l'intervention a été, à tort ou à raison, considérée comme un défi lancé au monde arabe et, au-delà, au Tiers-Monde. L'amalgame qui a été fait entre l'invasion du Koweït et l'occupation israélienne des territoires palestiniens, explicable en partie par le sentiment que les sanctions prévues en cas du non-respect des résolutions de l'ONU changeaient selon la "tête de client", a continué de déterminer la vision d'une grande partie de l'opinion publique arabe même après la guerre. L'ampleur de la défaite militaire subie par l'Irak a également provoqué un sentiment de frustration. Autant l'opposition irakienne, dans sa presque totalité, a soutenu l'ingérence humanitaire et tenté de s'implanter dans la zone d'exclusion, autant de larges parts de l'opinion publique arabe non-irakienne l'ont perçue comme une nouvelle atteinte à la souveraineté arabe et une nouvelle tentative de morcellement. L'Irak a été considéré comme la première victime du "Nouvel Ordre Mondial" y compris par les intellectuels, "laïcs" et "démocratiques" qui l'ont salué pour avoir sauvé "l'honneur de l'arabité"7. Toutefois, toutes les tentatives de l'Irak en vue d'exploiter ces sentiments se sont avérées insuffisantes pour mettre en place une mobilisation "anti-impérialiste". Les Kurdes n'assistaient pas uniquement à une spectaculaire médiatisation de leur cas ${ }^{8}$. Cette expérience marquait aussi une amplification sans précédent du phénomène de la transfrontaliérité. Parallèlement à l'établissement d'une administration kurde, autrement dit, à la satisfaction de l'une des revendications minimales des Kurdes (l'autre étant le renversement de Saddam) et malgré leur refus de sécession, le Kurdistan d'Irak est devenu une entité coupée du centre irakien mais ouvert sur d'autres horizons. En effet, l'arrivée massive des Kurdes irakiens en Turquie et en Iran, lors de l'exode, ainsi que l'implantation des Kurdes de ces deux pays, parfois sous forme de groupes armés comme le P.K.K. (Parti des Ouvriers du Kurdistan, de Turquie) ou le K.D.P. (Parti Démocratique du Kurdistan, d'Iran) dans les territoires contrôlés par les Kurdes irakiens ont considérablement élargi et intensifié les échanges intra-kurdes. Ce double mouvement a contribué à une profonde modification de la perception de soi (on est passé de l'appartenance à l'irakité à l'appartenance à la kurdicité), même s'il a parfois entraîné des affrontements intra-kurdes dont le plus important a opposé le gouvernement kurde irakien et le P.K.K. en novembre $1992^{9}$. Les relations commerciales avec la Turquie, et dans une mesure limitée, avec l'Iran, ont changé les axes 
économiques, voire géographiques. On emprunte désormais la route menant vers la Turquie, et dans certains cas vers l'Iran, pour établir des contacts extérieurs, mais rarement celle de Bagdad. Le Kurdistan est devenu, surtout après l'embargo décrété par Bagdad à son encontre, plus dépendant de l'extérieur que du centre; la mise en place de médias, notamment de chaînes de télévision kurdes, transmettant presqu'en direct des émissions de chaînes occidentales a créé une ouverture sur le monde; enfin, la présence d'organismes humanitaires dont les actions sont rarement coordonnés entre elles, mais également avec le gouvernement kurde et les militaires turcs et occidentaux, a "fragilisé" la notion des frontières rigides, défendue par Bagdad. Il est évident que les Kurdes irakiens ne pouvaient faire face à une telle évolution, étant donné les structures archaïques du "Front du Kurdistan", prévues uniquement pour l'exil et comportant le risque d'émergence des milices ${ }^{10}$. L'établissement, en peu de temps, d'une autorité centrale relevait autant du souci d'asseoir une légitimité parlementaire que de la volonté de gérer cette situation. Pour les Etats du MoyenOrient, la Guerre du Golfe et la libération de facto du Kurdistan irakien ont exacerbé le conflit hégémonique, à peine dissimulé, qui les opposait. La Turquie et l'Iran, bien qu'appréhendant l'expérience d'une assemblée kurde élue et ses effets sur leurs propres Kurdes, ont néanmoins tenté d'être présents militairement et de contrôler l'accès à cette région. Mais cette évolution traduisait également leur incapacité à maîtriser la situation et à s'imposer comme arbitre ou garant. Cela est aussi vrai pour les capitales occidentales qui ne pouvaient prévoir une transformation aussi rapide. Il ne faudrait cependant pas chercher dans cette évolution la "main masquée" d'un quelconque complot. En effet, la politique occidentale consistait au début à écarter la carte minoritaire. Les diplomaties occidentales ont cependant préféré composer avec le fait accompli qui au moins, sur le plan international, les déchargeait d'un problème : l'instauration d'une autorité centrale kurde empêchait le vide du pouvoir que Bagdad ne pouvait de toute manière pas combler. La zone d'exclusion devenait en outre un bastion pour limiter le champ d'action de Saddam, en le contraignant "pacifiquement" à quitter le nord. L'Occident avait également le sentiment de s'acquitter ainsi de sa "dette" à l'égard des Kurdes et de donner une base éventuelle à l'opposition. Les Kurdes irakiens ont su, de leur côté, adopter une politique pragmatique, miser sur la carte turque, au détriment de l'Iran qui était leur allié pendant la première guerre du Golfe (1980-1988), et surtout, donner une expression politique au pluripartisme qui existait au sein de leur société. Ces facteurs ne pouvaient que soulager l'Occident, hanté au début par l'éventualité d'une avancée chi'ite. En outre, les Kurdes ont su refuser l'option sécessionniste, ce qui leur a permis de se légitimer également en tant qu'irakiens et rassurer les Occidentaux sur l'intégrité territoriale du pays. Enfin, la mobilisation kurde permettait aussi de réduire considérablement le nombre de soldats occidentaux engagés pour protéger la zone d'exclusion.

2 "Diplomatie parallèle" ou "diplomatie humanitaire" Pour comprendre la période qui commence au Moyen-Orient après la Guerre du Golfe, nous préférons utiliser l'expression de "diplomatie parallèle", une nouvelle procédure de règlement des conflits régionaux qui a vu le jour dans les années 80 , plutôt que l'euphémisme de "diplomatie humanitaire". Précisions d'emblée que cette expression nous place de plain-pied dans le domaine de la géopolitique ; elle nous révèle également la profonde crise des expériences d'Etat-Nation et l'amplification sans précédent des conflits ethniques ou, d'une manière générale, des contestations ethniques ou nationalistes au Moyen-Orient. Cette double crise dont la contestation kurde est un épiphénomène 
important, modifie nécessairement les règles du jeu diplomatique dans la région. En effet, les Etats du Moyen-Orient, y compris l'Irak, n'ont pas attendu la Guerre du Golfe pour reformuler un nouveau jeu régional qui sonnait le glas d'un statu quo dont désormais aucun d'entre eux ne se satisfaisait. Or la remise en cause du statu quo, l'un des critères, à notre sens, de l'échec de l'expérience d'Etat-Nation ${ }^{11}$, ne pouvait se faire sans la revalorisation de l'un des éléments qu'il envisageait précisément d'éliminer : en l'occurrence les groupes non-étatiques. L'utilisation de la carte kurde a été un enjeu déterminant de la première guerre du Golfe (1980-1988), tout comme la guerre du Liban, qui a éclaté dans des conditions fort différentes, a été le théâtre d'un usage intense de toutes les dynamiques communautaires. La deuxième guerre du Golfe a également montré que les Etats de la région ne pouvaient faire abstraction de cette donnée. Le prix de cette reformulation du jeu régional est premièrement la limitation de la notion même de "souveraineté" des Etats, deuxièmement la légitimation, fût-ce par l'implicite, d'acteurs qui ne sont pas définis comme tels par le droit international, troisièmement l'acceptation du principe de l'ingérence de l'ensemble des Etats dans la région et dans les affaires intérieures de chacun d'entre eux. Une fois le statu quo ébranlé, ce n'est plus le principe de cette intervention que les Etats contestent, comme le montre la guerre entre l'Iran et l'Irak, mais le fait qu'il devienne une arme politique et militaire comme une autre, aussi "légitime" ou "illégitime" que les actions militaires elles-mêmes. Chaque Etat tente, à l'inverse, de l'utiliser à son profit avec une rentabilité maximum, en investissant autant que possible la "minorité" des "voisins" avec qui il est en conflit, et en enrayant le succès de ses adversaires chez lui. Il est facile d'évaluer les conséquences d'une telle évolution: d'abord, à la suite des interventions concurrentielles de multiples Etats, chacun d'entre eux voit sa souveraineté limitée et réduite sur le plan régional ; ensuite, la non-maitrise de la violence à l'échelle régionale permet également aux groupes minoritaires d'élaborer leurs propres stratégies, souvent à court ou moyen termes. Seulement si ces stratégies peuvent dans certaines circonstances notamment lors d'un vide d'autorité, être porteuses de succès, ils en sont en rarement les maîtres. Les acteurs minoritaires ne parviennent à gagner qu'en utilisant la carte de la régionalité, et en réussissant à se doter de moyens financiers et militaires importants; ainsi d'acteurs locaux, ils accèdent au statut d'acteurs régionaux. Ils peuvent également, signe du dépassement de leurs frontières, mettre en place un réseau diplomatique plus ou moins efficace à l'échelle internationale et alerter l'opinion publique occidentale. Pour contrecarrer leur impact sur le plan "national", l'Etat se surmilitarise et devient "policier" à l'intérieur de ses frontières fragilisées tout en cherchant à fragiliser les frontières de l'adversaire. Il est aussi amené à accepter le principe, contraire à son idéologie fondatrice, d'accorder des privilèges exceptionnels à certains segments de la minorité afin de les rallier à sa cause et de répondre de l'intérieur à la contestation minoritaire, ce qui aboutit, bien entendu, à un morcellement de sa souveraineté. Dans le cas kurde, cela signifie que l'Etat tente d'un côté d'épargner les Kurdes de l'extérieur et de jouer sur cette carte pour fragiliser le "voisin", de l'autre, de transformer la guerre à l'intérieur de ses frontières en une guerre avant tout, intra-kurde ${ }^{12}$. Alors que les acteurs minoritaires tentent d'accumuler des atouts régionaux pour les monnayer sur le plan intérieur, "national", les acteurs étatiques cherchent à maîtriser la situation intérieure pour oeuvrer plus facilement sur le plan régional. Ainsi, pour les deux acteurs, la stratégie mise en oeuvre consiste à passer sans cesse du national au régional afin d'obtenir un gain convertible d'un plan sur l'autre Chacun tente également de devenir le maître de ce jeu. Cependant, étant 
donné la multitude des acteurs, il semble clair que, aucun acteur étatique ne pourra maîtriser la situation et enrayer les effets de contamination interne au sein de sa propre minorité et, que, par ailleurs, il lui est impossible de contenir l'affaire sur un plan régional et d'éviter par là même le passage à l'internationalisation des conflits et des enjeux. Le risque est alors grand de voir se produire des "effets pervers" pour les Etats régionaux . Cette "logique" explique effectivement pourquoi le Kurdistan d'Irak est devenu, au lendemain de la guerre, une entité quasi souveraine. La stratégie minoritaire, misant largement sur les atouts de la "régionalité" a pu, pour le moment du moins, mettre à son profit les faiblesses et les intérêts pragmatiques des acteurs étatiques, régionaux ou internationaux, pour prendre le dessus et s'imposer comme le pouvoir de fait au Kurdistan. Les Kurdes sont loin, cependant, de maitriser la situation, car leur stratégie ne peut être porteuse qu'aussi longtemps que les faiblesses ou les intérêts des autres, valorisant l'usage de la carte kurde, se perpétuent et que les capitales occidentales préfèrent un état de fait plutôt qu'une collaboration avec Bagdad, autrement dit, aussi longtemps que le vide qui résulte des intérêts conflictuels des acteurs étatiques leur permet de se maintenir.

Pourquoi "l'intervention humanitaire" a-t-elle réussi au kurdistan d'Irak ? Après le Kurdistan d'Irak, d'autres interventions humanitaires ont été décidées par des instances internationales, en Somalie, au Cambodge et dans l'ex-Yougoslavie. Là aussi, l'humanitaire était lié, ou était issu, de la dimension plus large d'un processus conflictuel, incluant divers acteurs et surtout, divers degrés de violence. La sensibilisation de l'opinion publique ${ }^{13}$, la médiatisation, l'expérience de terrain des ONG, etc. ont joué un rôle certain dans la décision d'intervenir au Kurdistan, comme ailleurs par la suite. Le cas kurde n'est donc pas unique, mais il a une singularité : c'est le premier cas où l'ingérence humanitaire, prônée jusque là par les ONG seules, a pu dépasser la notion de "l'accès aux victimes" pour se doter d'une légitimation internationale. La résolution 688 faisait-elle pour autant figure de jurisprudence? Il faut certes admettre que le cas kurde a au moins un dénominateur commun avec les autres opérations humanitaires: on y assiste à une politique d'ajournement, de "suspension" du conflit où les volte-face sont toujours possibles en fonction de l'évolution des rapports de force et des intérêts des Etats de la région. Cette politique permet aussi aux Etats-Unis de se projeter et de se présenter comme un élément vital, une nécessité dans la région. De même, la Turquie, acteur régional, a intérêt, sur une longue période, au prolongement de la politique d'ajournement. Pourtant, rien n'est joué, le retour en force de Saddam Hussein comme leader incontournable et internationalement reconnu de l'Irak n'est pas à exclure. En effet, la tension entre l'Iran et l'Occident, ou entre l'Iran et le monde arabe, peut bel et bien aboutir à une nouvelle reconnaissance de l'autorité ba'athiste sur l'ensemble de l'Irak. Malgré ce dénominateur commun de "suspension" du conflit, il est néanmoins difficile de prendre le Kurdistan d'Irak comme un laboratoire pour expliquer le "processus d'intervention humanitaire", car les facteurs qui séparent cette expérience des autres sont nombreux et importants : d'abord, le Moyen-Orient, de par ses richesses pétrolières, est vital pour l'Occident ce qui n'est naturellement pas le cas de la Bosnie. Dans le cas kurde, il était possible de mobiliser d'importantes ressources humaines sans rencontrer de grands risques, alors qu'en Bosnie, toujours à titre d'exemple, la situation est bien plus compliquée et les risques de pertes humaines ${ }^{14}$ sont élevés, tout comme sont élévés les risques de se trouver parti prenante dans la "balkanisation" en pleine Europe. Il est aussi évident qu'une telle éventualité ne manquera pas d'accentuer davantage les 
brèches au sein de la "politique étrangère" de la Communauté européenne. Au Kurdistan irakien, il était facile de désigner les parties et de différencier le "bourreau" de la "victime", bien entendu, mais aussi, de choisir entre le "bourreau" et le Conseil de Sécurité en tant qu'adversaires, et donc de trancher, ce qui n'est nullement le cas en Somalie par exemple, ou voire même au Cambodge. Si dans le cas des Kurdes, l'une des parties, Bagdad était militairement vaincue et désignée, en quelque sorte, comme l'ennemi, en revanche, les Serbes, par exemple, sont considérés comme une partie belligérante, et non comme l'adversaire direct de l'ONU. Dans le cas kurde, il était facile de créer un consensus entre les cinq membres permanents du Conseil de Sécurité, alors que la Russie menace d'utiliser son droit de veto concernant la Serbie et que la Chine en fait autant à propos du Cambodge. La mauvaise conscience, née de l'abandon des populations irakiennes à leur propre sort lors de l'insurrection après les avoir encouragées à se révolter contre la "dictature de Bagdad", facilitait la mobilisation de l'opinion publique dans le cas du Kurdistan d'Irak, au point qu'elle devint un facteur de decision making, ce qui n'est naturellement pas le cas pour la Bosnie, même si les scènes retransmises en direct par les chaînes de télévision sont plus tragiques. En outre l'opinion publique est indifférente et se résigne plus facilement à une vision fataliste ( « de toute manière, ils ne désirent que s'entre-tuer... pour quelle raison devons-nous nous impliquer dans des "conflits tribaux" 15 ou "une guerre de religions" »). Ce fatalisme de l'opinion publique consiste, au mieux, à attendre des pouvoirs qu'ils agissent et ne vise nullement à les pousser à l'action comme elle l'avait fait dans le cas kurde. Les Kurdes irakiens ont pu prendre la situation en main et créer une administration qui fonctionne, capable de gérer le quotidien, de se substituer à l'autorité centrale de Bagdad, et d'empêcher ainsi le vide du pouvoir, ce qui n'est pas le cas en Somalie ou au Cambodge, où on est en face, non pas d'une minorité linguistique ou ethnique en rébellion contre le pouvoir central, mais de factions en lutte. Les difficultés financières, devenues plus aiguës avec l'envoi de casques bleus en Somalie, au Cambodge et en ex-Yougoslavie (augmentant par là même le déficit budgétaire de l'ONU qui atteint 4 milliards de dollars) semblent constituer également une raison poussant l'Occident à agir de façon minimum dans ces pays. La stratégie kurde ellemême a aidé au succès de l'humanitaire. En effet, les Kurdes ont, au début, mal apprécié le terme même "d'humanitaire" et surtout le fait d'être réduits à la condition de nécessiteux. Ils ont également rapidement surmonté "l'humanitaire" strictement parlant pour envisager de se constituer en interlocuteurs crédibles, reçus par John Major, François Mitterrand, Al Gore etc., et pour commencer leur reconstruction économique, notamment agricole. Ils se sont aussi montrés remarquablement pragmatiques par rapport à l'appartenance à l'Irak ${ }^{16}$. En effet, la sur-médiatisation de leur problème a poussé les Kurdes à cesser de jouer eux-mêmes la carte "victimaire" ${ }^{17}$. Ils ne pouvaient d'ailleurs plus utiliser une telle stratégie, car au-delà d'une certaine limite admissible de martyrologie, destinée à l'opinion publique kurde, cette fuite en avant n'était pas désirée par la population qui aspirait intensément à la stabilité. Ces mêmes aspirations expliquent que, malgré l'indignation de l'opinion publique internationale, des chefs kurdes aient tenté d'apaiser la situation, allant jusqu'à embrasser Saddam et chercher la moins catastrophique des paix avant d'interrompre les négociations avec Bagdad. Enfin, contrairement au sud chi'ite, le Kurdistan ne faisait pas peur aux Occidentaux. Hanté par une nouvelle révolution islamique, l'Occident préférait encore un Saddam affaibli à une prédominance iranienne. Alors que les chefs kurdes se sont détachés à temps de la suprématie iranienne, ont établi des 
liens diplomatiques avec les capitales occidentales, la spontanéité et l'isolement de la révolte au sud n'ont pas laissé aux chi'ites le temps de développer un projet antiSaddam, encore moins de convaincre l'Occident ${ }^{18}$. Contrairement aux Chi'ites, mais aussi, aux musulmans de Bosnie, les Kurdes pouvaient être présentés comme de "bons musulmans", modérés et laïcs. Les Kurdes irakiens ont su tirer profit de l'ensemble de ces atouts. La modération de leurs revendications et l'auto-légitimation de soi en tant que Kurdes et Irakiens en même temps, leurs efforts de médiation et de pondération à l'extérieur du Kurdistan irakien ${ }^{19}$, se sont avérés fort utiles et les ont relevés au rang d'interlocuteurs crédibles. Ainsi, contrairement aux autres cas "humanitaires", le Kurdistan irakien a bénéficié par le concours d'une série de circonstances favorables, de cartes maitresses. Mais tous ces atouts réunis auraient pu néanmoins ne pas suffire. En effet, si cette expérience peut être considérée comme particulière, c'est qu'elle est inscrite aussi pleinement dans le jeu de la géopolitique régionale, dans ce que nous avons qualifié de "diplomatie parallèle". Parmi les facteurs qui lui ont permis de durer, on ne saurait perdre de vue le rôle joué par la Turquie. En effet, Ankara qui avait supporté le poids des flux migratoires kurdes irakiens à la suite des opérations d'Anfal en 1988 et d'une manière plus massive à la suite de la guerre du Golfe en 1991, avait été aussi la première capitale à demander l'intervention de l'ONU et la création de zones d'exclusion au Kurdistan d'Irak. Or, on pouvait difficilement soupçonner la Turquie d'être mue par des sentiments pro-kurdes et il est pour le moins évident que dès le départ, malgré la charge extrêmement lourde que représentait ces réfugiés, le problème kurde irakien ne constituait pas uniquement, voire essentiellement pour ce pays une question humanitaire. Il a été et reste, au contraire, partie intégrante de sa principale difficulté interne et externe qui perdure depuis près de 70 ans. Le problème kurde suscite, dans l'esprit de nombreux dirigeants turcs, une vision de cauchemar : le démembrement, dont le Traité de Sèvres, prévoyait en outre la création d'une Arménie et d'un Kurdistan. Comment expliquer alors ce revirement et surtout ce paradoxe consistant à mettre en oeuvre une politique intérieure extrêmement rigide à l'égard de ses propres Kurdes toute au long de l'année 1992 et une politique de "protection" à l'égard des Kurdes de l'Autre? La Turquie a été, pour des raisons évidentes, toujours sensible à ce qui se passait chez les "Kurdes des autres". Dès son émergence, dans les années 1920 , le problème kurde est un problème transfrontalier, entravant plus d'une expérience étatique au Moyen-Orient. Autrement dit, un mouvement kurde "local" peut facilement contribuer à influer sur la situation dans les autres pays. Les révoltes kurdes de l'époque kémaliste en Turquie (1925-1938) ont eu un impact direct aux Kurdistans irakien et iranien. Par la suite, pour diverses raisons, le Kurdistan irakien a joué un rôle capital dans l'évolution des Kurdistans de Turquie et d'Iran. La république de Mahabad (Iran, 1946) a été possible grâce à l'intervention des Kurdes d'Irak; la révolte de Moustafa Barzani en 1961 a eu un effet mobilisateur immédiat sur les Kurdes de Syrie et d'Iran, mais surtout sur ceux de Turquie et a touché non seulement les tribus frontalières, mais aussi les intellectuels, représentant ainsi un véritable facteur de la renaissance du nationalisme kurde qu'on croyait mort depuis 1938. Plus récemment, l'arrivée de plus de 100.000 Kurdes de Bahdinan en 1988, a provoqué une mobilisation importante en Turquie. La Conférence Internationale de Paris, organisée par l'Institut Kurde de Paris en 1989, pour dénoncer, entre autre, l'usage des armes chimiques au Kurdistan d'Irak a également abouti à une vaste mobilisation en Turquie et a provoqué la division de la principale formation social-démocrate, le S.H.P. Ces événements et la radicalisation qui a suivi, ont détruit la notion des frontières dans la 
mentalité kurde de Turquie. Last but not least, la présence de Kurdes de Turquie en Irak et les rapports traditionnels entre les Kurdes d'Irak et ceux de la région frontalière n'ont pas manqué de traumatiser le pouvoir d'Ankara. Ce survol historique explique clairement pourquoi, pour Ankara, le problème de "l'Irak du Nord" comme le dénommaient timidement les politiciens turcs, ne pouvait être uniquement de nature humanitaire. Ankara ne pouvait prendre cette position ,"humanitaire", pour les mêmes raisons que la Grande-Bretagne ou la France, par exemple, même si, dans leur cas également, l'existence d'arguments non-humanitaires ne faisait guère de doute. On pouvait en effet s'attendre à ce que la Turquie, en 1988 comme en 1991, répète le même scénario que celui de 1975 et décide de fermer ses frontières aux Kurdes irakiens. On pouvait également penser qu'une fois encore, elle donnerait un soutien, sinon franc, du moins voilé à Bagdad. Or, avant, et surtout après l'établissement des zones de sécurité, l'attitude turque fut pour le moins surprenante, d'autant plus qu'Ankara avait mis Bagdad en garde contre de nouvelles attaques visant les Kurdes, et avait publiquement envisagé la possibilité d'une fédération kurdo-arabe à ses frontières du sud. Ajoutons à ces facteurs, de nombreux changements concernant directement la Turquie sur la scène internationale depuis la guerre du Golfe. La fin de l'empire soviétique concernait Ankara au tout premier plan. Or, l'Azerbaïdjan ainsi que les autres républiques turciques ont été déçues par la passivité d'Ankara. Plus récemment, Ankara a haussé le ton à l'égard d'Erevan, tout en avouant sa complète incapacité à intervenir effectivement dans le conflit qui l'oppose à Bakou. De même, la guerre civile et le démembrement de la Yougoslavie n'ont pas manqué d'accroître la valeur utilitaire de la Turquie. Mais là également, à la grande déception des Bosniaques, la Turquie s'est plutôt contentée de déclarations non suivies d'effet. Dans cette période et cette aire de turbulence régionale, le Kurdistan irakien a continué d'occuper la place centrale dans la politique extérieure turque. De 1991 à la fin de 1992, la Turquie a organisé contre les positions de P.K.K. au Kurdistan irakien des dizaines d'interventions militaires dont celle de novembre 1992, qui semble, a posteriori, avoir été décisive. Les réunions tripartites entre Ankara, Damas et Téhéran sur le sort du Kurdistan irakien ont été bien plus importantes pour la diplomatie turque que celle, tenue à Istanbul, sur l'Asie centrale. Peut-on d'ailleurs uniquement parler de politique étrangère? Rien n'est moins sûr. En effet, le problème kurde a constitué, depuis la guerre du Golfe, le point central des politiques internes et militaires d'Ankara. Si l'on assiste à une ingérence "humanitaire" et militaire d'Ankara au Kurdistan d'Irak, à l'inverse, les Kurdes irakiens eux-mêmes n'ont pas hésité à intervenir sur la scène politique turque sur des thèmes concernant les Kurdes de Turquie eux-mêmes. On ne peut d'ailleurs plus parler d'une simple interaction entre ces politiques, mais plutôt d'une symbiose totale de ces divers sujets, voire des politiques interne et externe.

La politique étrangère turque à l'égard de l'Irak et du Kurdistan d'Irak L'humanitaire peut assurément devenir un instrument au service de la géopolitique. Les rapports ambigus qui gèrent le passage de l'un à l'autre ne sont pas faciles à saisir, surtout dans le cas des pays du Moyen-Orient, directement concernés par le théatre des opérations. Le cas turc est encore plus compliqué car "l'humanitaire", tel que l'entendent du moins les ONG y est presque absent en tant que souci. Pour comprendre dans cet exemple le passage de la géopolitique à la position "humanitaire" et le retour ensuite à la géopolitique, il nous semble nécessaire d'analyser brièvement les trois phases de la politique turque à l'égard de l'Irak et du Kurdistan d'Irak. La première période que nous pouvons ouvrir par la signature du traité d'Ankara en 1926 fixant 
"définitivement" le sort de la région pétrolifère de Kirkouk/Moussoul est caractérisé par le statu quo avec pour règle d'or la reconnaissance des seules relations d'Etat à Etat. L'objectif du statu quo se résume au contrôle collectif des frontières, à la maîtrise du mouvement kurde à l'échelle régionale et à l'établissement d'un système de sécurité collective. Les documents de base de cette politique régionale se trouvent, entre autre, dans les traités anglo-irako-turc de 1926, de Saadabad (1937) et de Bagdad (1955). La Turquie a poursuivi cette politique pendant les décennies suivantes, y compris lors de la révolte de Barzani (1961-1975) ; lors de la répression de cette révolte, la Turquie a refusé d'ouvrir ses frontières aux rescapés kurdes. A la fin des années 70, Ankara et les organisations kurdes d'Irak ont établi certaines relations, mais globalement la même politique a été maintenue. La visite du futur président turc, général Evren, alors Chef d'état-major, à Bagdad en 1979 ne fit que renforcer la politique de sécurité collective. En 1983, 1984 et 1987 la Turquie est intervenue à trois reprises au Kurdistan irakien où le contrôle de Bagdad était battu en brèche par les avancées des troupes iraniennes et des forces militaires kurdes. L'objectif de ces opérations, légalisées sur le plan juridique par le Traité d'Ankara signé en 1984, était de poursuivre les membres du P.K.K., mais également de limiter l'influence des Kurdes irakiens et donner un avertissement aux autorités de Téhéran. A la fin des années 80 cependant, nous observons une transition de la logique du statu quo et de la sécurité collective à un jeu de "grandes puissances" dans la région. En effet, à partir de cette décennie, les Etats, devenus suffisamment forts, s'offrent désormais le luxe d'avoir des ambitions régionales, ce qui brise bien entendu les tabous concernant les frontières, mais surtout, augmente considérablement l'importance de la carte kurde dans la région. La guerre entre l'Iran et l'Irak était la première grande manifestation, échappant au contrôle des protagonistes d'ailleurs, de ce changement radical ; la politique régionale de la Syrie, allant jusqu'à soutenir les Kurdes de Turquie et d'Irak, ainsi que la menace turque d'utiliser l'eau comme une arme, constituaient d'autres signaux de "dérapages". En outre, la Turquie a envisagé, en 1987-1988, en accord avec le Pentagone, la possibilité d'envahir le Kurdistan irakien pour barrer la route à Téhéran ${ }^{20}$. La deuxième période s'étend de 1986 au 16 janvier 1991. Vu d'Ankara, elle est marquée par la fin officielle du statu quo avec l'Irak, concrétisée en mai 1990 par la visite d'Etat du Premier ministre turc Y. Akbulut à Bagdad. La visite, et surtout la rencontre entre Saddam et Akbulut se sont soldées par un véritable scandale diplomatique, à tel point que le président irakien a refusé, sans la moindre formule diplomatique, l'invitation de son homologue turc. Deux points ont montré le profond désaccord entre les deux capitales : concernant les eaux, Saddam Hussein demandait un accord tripartite (Syrie, Irak, Turquie) préalable à la normalisation des relations et soutenait les revendications syriennes sur les eaux de l'Euphrate; concernant la demande d'Ankara d'avoir le droit d'intervenir militairement au Kurdistan irakien pour poursuivre les militants du P.K.K. qui y avait établi leurs bases, la réponse fut une fin de non recevoir. Bagdad faisait comprendre que la sécurité de son voisin, souci majeur dans une logique de statu quo, ne lui importait guère ou, du moins, dépendait de certaines conditions ${ }^{21}$. D'autres facteurs sous-jacents, tels que la reconnaissance par Ankara, de la souveraineté du P.D.K. irakien sur les Kurdes réfugiés en Turquie n'étaient pas non plus absents. Cette visite a permis à la Turquie de réaliser que la fin de la guerre entre l'Iran et l'Irak était loin de permettre un retour aux bonnes vieilles méthodes d'antan. Ce qui explique pourquoi pendant, et surtout, après la guerre, la Turquie eut une position de chef de file intransigeant. En effet l'attitude d'Ankara s'expliquait certes par son engagement pro- 
occidental, mais également par l'existence de la tension, née de la fin du statu quo; cette tension impliquait bien entendu, la peur, mais elle permettait en même temps, à Ankara, avec le droit de réciprocité, d'envisager de nouvelles perspectives régionales. Le président turc Turgut Özal avait réalisé que le moment de ce changement était venu. Son pragmatisme l'a poussé à abandonner l'héritage kémaliste, ne reconnaissant que les Etats comme acteurs légitimes. Il a levé aussi l'hypothèque de la sacralité des frontières puisqu'il a explicitement envisagé leur possible remise en cause. Sa stratégie visait à utiliser la carte américaine afin de faire de la Turquie la superpuissance régionale. Ses discours à l'époque, ainsi que les textes de ses proches sont suffisamment clairs sur point ${ }^{22}$. La politique d'Özal a connu cependant un succès mitigé. En effet, plusieurs conditions étaient nécessaires pour qu'elle puisse parvenir à ses fins, notamment que la majeure partie des forces politiques se range derrière lui. Or , cellesci ne l'ont en grande partie nullement soutenue, et surtout l'armée s'est déclarée hostile à toute intervention militaire turque, pour empêcher entre autre l'émergence d'un acteur kurde irakien ou une nouvelle révolution islamique. Le soutien américain lui-même a été limité et surtout réservé. La Maison Blanche n'avait pas de politique clairement définie. En outre, l'opposition affichée de la Syrie, de l'Irak et de l'Arabie saoudite était trop importante pour que les Etats-Unis donnent carte blanche à Özal. Enfin, il n'est pas sûr que Washington ait été prêt à accepter de faire de la Turquie son seul cheval de bataille dans cette région troublée. La troisième période qui débute avec la crise et la guerre du Golfe, est marquée par la révolte kurde qui éclate en mars, la répression et l'exode qui suivent. Özal a été le premier homme d'Etat à demander alors, avec la France, la création d'une zone de protection pour les Kurdes, dont l'inviolabilité serait garantie par une armée de la coalition. Au début, sa suggestion a été fraîchement accueillie par la Maison Blanche, qui a cependant cédé face à la pression des capitales occidentales, de sa propre opinion publique et surtout face à l'ampleur de l'exode. De même, d'une manière surprenante, Özal a secrètement reçu Talabani à Ankara et rendu publique cette "affaire" avant même d'être invité à le faire par les journalistes. Cette visite surprise a été suivie par des rencontres avec Barzani, par l'ouverture de bureaux des partis politiques kurdes à Ankara, et surtout des premières déclarations d'Özal sur la possibilité d'une fédération kurdo-turcomano-arabe en Irak. Devant la faiblesse à la fois numérique et politique des Turcomans cet élément a été abandonné cependant pour laisser place à l'idée d'une fédération kurdo-arabe. Avec le changement de gouvernement en novembre 1991, Özal a perdu la maîtrise de la politique étrangère, même s'il est resté, jusqu'à sa mort en avril 1993, l'interlocuteur incontournable de tous les dirigeants kurdes de passage à Ankara. Mais le nouveau gouvernement, bien que plus prudent en ce qui concerne la politique à suivre à l'égard des Kurdes, n'hésite pas non plus à les recevoir au plus haut niveau. Sa politique est déterminée par plusieurs facteurs : l'absence de l'autorité centrale de Bagdad lui laisse toute latitude pour gagner la suprématie militaire dans le nord de l'Irak, à la fois par rapport à son voisin du sud que par rapport au P.K.K., voire par rapport à l'Iran. Les risques d'un front tacite irako-syrien concernant les contentieux sur l'eau sont également écartés. Plus important encore, si le pays nie avoir une politique irrédentiste, consistant à récupérer l'ex-province ottomane de Kirkouk, l'une des régions pétrolifères les plus riches du monde, les portes restent grand ouvertes à une sorte de protectorat turc au Kurdistan d'Irak. Mais pour de telles perspectives et occasions historiques Ankara devait naturellement payer un prix, notamment, donner, en contrepartie, quelque chose de tangible ou, du moins, certaines garanties aux Kurdes. De l'ouverture des 
frontières et l'acheminement des vivres, à la reconnaissance, de facto, de l'administration kurde, des élections, voire, de l'Etat fédéral, il n'y avait qu'un pas. En effet, si la Turquie a refusé de reconnaître l'Etat fédéré kurde auto-proclamé, elle n'est cependant pas allée jusqu'à le condamner ou changer sa politique à l'égard des Kurdes d'Irak. De même, si les réunions entre Ankara, Téhéran et Damas ont bel et bien envisagé des mesures à l'encontre d'un éventuel irrédentisme kurde, elles n'ont pas pour autant abouti à la mise en place d'un nouveau statu quo, et c'est, curieusement, Washington qui a renouvelé en Avril 1993, le message de confiance d'Ankara destinés aux Kurdes du Sud. Ainsi, l'appui d'Ankara devenait la condition première de la réussite de l'expérience humanitaire au Kurdistan d'Irak, à la fois en ce qui concerne l'acheminement de l'aide et l'installation de la force de déploiement rapide chargée de protéger les Kurdes, que sa transformation en une entité plus ou moins séparée de l'Irak, surtout par la reconnaissance des douanes et des postes kurdes à sa frontière ainsi que la formation d'une armée kurde unifiée. Le pragmatisme et les contraintes, tout comme les ambitions régionales, ont déterminé la position de la capitale turque qui a su combiner, tout au long de 1992 une violence interne intense avec une politique "d'amitié" pour les Kurdes du sud. Conclusions Dès lors que nous nous plaçons dans le domaine de la géopolitique, nous sommes également confrontés aux questions suivantes : quel est l'Etat interessé et quel est usage qui est fait de la carte kurde ? En effet, les Kurdes ne préoccupent pas un seul Etat et l'usage de cette carte n'est nullement l'apanage d'un seul d'entre eux. Dans leur cas, plus que dans d'autres, nous constatons l'élargissement $\mathrm{du}$ fait transfrontalier et l'établissement de nouvelles matrices dans les relations internationales. Le processus de "l'intervention humanitaire" qui continue encore de nos jours au Kurdistan d'Irak ${ }^{23} s^{\prime}$ inscrivait dans la diplomatie parallèle et accentuait jusqu'à ses suites logiques et inévitables la brèche qui était apparue au début de la décennie 80 dans les relations interétatiques au sein de la région. On ne s'étonnera donc pas de voir que certaines des remarques qui ont retenu notre attention ne concernent que marginalement "l'humanitaire" ; celles-ci, d'ordre géopolitique, expliquent au contraire, pourquoi le retour à un statu quo, règle d'or d'un système de sécurité collective des Etats, n'est désormais plus possible, mais elles nous mettent également en garde contre tout pronostic concernant l'avenir. Certes, l'on peut prévoir qu'une mobilisation à l'échelle planétaire dans la région, comme lors de la guerre du Golfe, est difficile à répéter. Il n'en reste pas moins que les conflits régionaux ne peuvent être résolus par "l'humanitaire" ; ils demeurent et risquent de s'amplifier à l'avenir. Il convient, avant toute autre chose, de constater que l'ouverture de la boîte de Pandore qu'est le Moyen-Orient change nécessairement les données classiques de la région. Non seulement l'incapacité des grands Etats à contrôler les petits, fait visible déjà à l'époque de la bipolarité, se traduit d'une manière de plus en plus évidente de nos jours, libérant ainsi de gigantesques potentiels d'affrontements, mais les données relatives à la violence à l'échelle régionale changent considérablement. C'est, avant tout, la violence étatique qui s'amplifie pour dépasser la simple coercition interne à mesure qu'elle étire toujours son champ de manoeuvre externe. A une plus grande échelle, elle vise également les autres Etats. En contrepartie cependant, cette violence étatique peut-être amenée à ménager les membres d'une minorité donnée en dehors de ses frontières et l'utiliser comme une carte efficace contre les autres capitales. Cela n'empêche pas cependant l'acteur minoritaire de mettre également en place des stratégies autonomes, par le biais soit des instruments de la violence soit des instruments diplomatiques ou de leur combinaison. Deuxièmement, la diplomatie peut 
s'avérer insuffisante pour régler les problèmes qui découlent de cet éclatement à savoir les tensions interétatiques, comme le montrent la guerre entre l'Iran et l'Irak ou l'invasion du Koweït, mais également celles relatives à des conflits minoritaires. L'introduction de la diplomatie parallèle peut alors tenter d'apporter des "solutions alternatives", mais nécessairement à court terme. Force est de constater que la carte minoritaire risque de jouer un rôle capital dans l'évolution de ces conflits. La guerre Iran-Irak s'est transformée en grande partie en une lutte autour du Kurdistan d'Irak. La stratégie turque, depuis la crise du Golfe, s'explique largement par la volonté de miner l'influence de Téhéran au Kurdistan d'Irak et d'écarter l'Irak comme éventuel chef de file d'une domination arabe dans la région. La réponse iranienne à la stratégie turque s'est traduite par un soutien à peine voilé au P.K.K., pendant sa guerre contre les Kurdes irakiens. De même, depuis quelques mois, Téhéran n'hésite pas à intervenir militairement dans le Kurdistan irakien, officiellement pour poursuivre les guérilleros kurdes iraniens. Une aggravation de la tension entre l'Occident, la Turquie et l'Iran peut, de nouveau, vivement réactualiser les cartes kurdes en Turquie et en Iran, et augmenter considérablement la valeur stratégique du Kurdistan d'Irak. Troisièmement, la résolution 688 a couronné l'action des ONG tout en la dépassant largement, en faisant de l'humanitaire une action politico-militaire, susceptible d'être appliquée si les conditions requises sont réunies. Mais outre que l'ensemble des conditions requises peut rarement être réunies comme c'était le cas pour le Kurdistan irakien, il serait singulièrement naïf d'imaginer que cette résolution a substitué l'humanitaire à la politique classique d'Etat. En contrepartie, il serait pour le moins absurde de chercher une quelconque rationalité, visant un quelconque plan de domination ou de partage du Moyen-Orient de la part de "l'Occident impérialiste". Une telle tentative de rationalisation des politiques d'Etat risque de réserver aux observateurs des surprises encore plus inattendues que la guerre du Golfe. En effet, l'absence de politique tout comme la politique de pis-aller peuvent parfois expliquer les véritables volte-face des puissances. Par ailleurs, "l'humanitaire" reste juridiquement difficile à encadrer. Comme le précise M.-C. Smouts, "le droit international est fait par les Etats et pour les Etats. Il est rarement sûr et se modifie selon les rapports de forces"24. En effet, il ne faudrait pas perdre de vue que la demande même d'accéder aux victimes, la revendication initiale des ONG inclut, fût-ce implicitement, la reconnaissance du droit des Etats à "victimiser". Mais au delà cette constatation de base, il est aussi vrai que, même lorsqu'il est appuyé par une force militaire et une mobilisation planétaire afin de "couper la main au bourreau", pour paraphraser B. Kouchner, l'humanitaire se trouve indéniablement dans l'incapacité de régler des conflits ethniques dont la solution semble nécessiter une nouvelle interprétation $d u$ droit et du politique, et le dépassement de l'Etat-Nation comme la seule forme d'existence humaine. En effet, le véritable noeud du problème semble se trouver dans l'impossibilité d'implanter l'EtatNation ailleurs que sur sa terre natale, et dans l'incompatibilité, concrétisée par un discours nationaliste à outrance, entre ce modèle et la nature "mosaïque" d'une région. Cette incompatibilité ne permet pas seulement à des groupes communautaires, voire claniques d'accaparer le pouvoir au détriment des "sociétés civiles" et au mépris de la lettre même des constitutions, comme nous l'observons souvent au Moyen-Orient, mais elle fait aussi dépérir la notion de frontière. Les contestations minoritaires elles-mêmes reflètent l'échec de ce modèle au Moyen-Orient, y compris lorsqu'elles formulent à leur tour la demande de sa reproduction à une plus petite échelle. La gravité de la crise que le Moyen-Orient vit actuellement semble souligner la nécessité de reconsidérer 
également la notion des frontières et d'envisager de "dénationaliser" l'Etat. Cela nous renvoie à la nécessité d'inventer des formes politiques et juridiques qui, sans remettre en cause les entités étatiques, permettraient aux minorités, de s'insérer dans une sphère publique et de jouir, en même temps, de droits extra-territoriaux ou transfrontaliers lorsque le groupe est divisé entre plusieurs Etats. Ces droits peuvent inclure la mise en place de certaines instances collectives, telles que des activités culturelles, des liens administratifs officialisés ainsi que la gestion des espaces économiques. De telles mesures sont susceptibles de satisfaire les minorités, de donner une légitimité aux Etats et de réduire les risques d'affrontements entre eux. L'action humanitaire, même lorsqu'elle est réussie, ne saurait être un remède à cette crise et ne fera qu'ajourner les problèmes. En effet, alors que les illusions sur d'un nouvel ordre mondial se sont révélées vaines, force est de reconnaître que les alliances internationales nécessaires pour organiser une action humanitaire à l'échelle planétaire ne pourront se constituer que rarement et avec bien des difficultés et ce, même dans un monde post-bipolaire. Il serait en effet illusoire de penser que la disparition des blocs conduira automatiquement aussi à l'homogénéisation et l'humanisation des actions, et surtout des stratégies et des intérêts étatiques ${ }^{25}$. Or, qui dit innovation juridique dit aussi retour aux fondements mêmes de la philosophie politique et, aux soucis de la "paix perpétuelle" alors que les acteurs et les enjeux, étatiques ou non, ne cessent de se multiplier. Une fois de plus au cours de l'histoire humaine, le domaine politique semble être à la croisée des chemins entre la morale (et l'invention volontariste) et la raison d'Etat. Quant à l'humanitaire, il semble être capable de se perpétuer comme l'appendice de l'un ou de l'autre, avec une formidable capacité de passer de l'un à l'autre. Il ne semble, en tout cas, nullement constituer une troisième alternative.

\section{NOTES}

1. Plus de deux ans après la Guerre du Golfe qui, semble aujourd'hui, être tombée dans l'oubli, nous traiterons ici l'un de ses aspects : l'aspect dit humanitaire. Il est évident cependant qu'au delà de cette dimension, au-delà aussi des pertes humaines importantes, cette guerre a suscité de nombreuses questions, d'ordre éthique, telles que la responsabilité du système international dans le renforcement du régime de Saddam Hussein, la question de la démocratie, du pluralisme politique dans le monde arabe, des droits des minorités, de l'universalisme et des expériences politiques etc. Que l'on ne nous tienne pas rigueur d'avoir délaissé l'ensemble de ces sujets sans lesquels la discussion sur l'humanitaire elle-même ne saurait qu'être mutilée. Nous nous permettrons cependant de renvoyer le lecteur à l'excellent numéro collectif des revues Esprit et Les Cahiers d'Orient sur ces sujets (Juin 1991, 348 p.).

2. C'était surtout Washington qui se faisait le défenseur de la "neutralité", alors que les autres capitales, Paris notamment, demandaient une intervention.

3. Les données économiques montrent clairement que Bagdad a perdu le contrôle du centre au Kurdistan : lors des échanges économiques avec l'extérieur le cours d'un 
dollar, qui était de 65 dinars en Irak (voire, parfois, même 90 dinars) n'était que de 25 dinars au Kurdistan. Les commerçants refusaient d'accepter les billets de 50 ou 100 dinars que Bagdad a mis en circulation après la Guerre ou ne les acceptaient qu'au dessous de leur valeur affichée ( 30 dinars pour des coupures de 50, par exemple). Pour obliger, entre autres raisons, le gouvernement kurde à céder, Bagdad a retiré de la circulation des coupures de $25 \$$. Cette mesure a provoqué une véritable panique et a poussé les dirigeants kurdes à envisager soit de créer une monnaie kurde, soit de demander à intégrer les kurdes dans le système monétaire turc.

4. Qui interdit le survol par l'aviation irakienne de la zone située au nord du 36ème parallèle.

5. Libération, 22.avril 1991.

6. Kouchner (B.) : "c'est quoi l'humanitaire ? C'est d'abord une indignation. Ensuite une obstination, et peut-être une méthode. La France à ce sujet a fait beaucoup, mais à l'intérieur des mandats des Nations Unies, mandats successifs et insuffisants mais qui vont se préciser. La France a fait beaucoup mais nous ne pouvons pas prétendre avoir tout réglé. La politique doit apprendre de l'humanitaire, pas le contraire. Elle apprend. C'est lent", cité in Le Monde des Débats, n5, février, 1993.

7. Cf. sur ce sujet le texte de Zeghidi (S.), "De la maturité de la pensée à la dérive politique. Lettre ouverte à Hichem Djait", Esprit, Les Cahiers de l'Orient, Juin 1991, pp. 32-52.

8. Malgré les cris d'alarme des "French Doctors", la guerre chimique de Saddam au Kurdistan à partir de 1988 n'avait suscité, somme toute, que de faibles échos dans la presse occidentale.

9. Mais il est question, depuis l'échec du P.K.K. dans cette guerre, d'une réconciliation et de la possibilité que le chef de ce parti s'installe au Kurdistan d'Irak. 10. Le "vide" du pouvoir a libéré un formidable potentiel militaire non contrôlé. D'anciennes brigades alliées au pouvoir se sont converties au "nationalisme" et ont rejoint les rangs des partis politiques sans dépendre cependant d'un contrôle quelconque. La peur de voir émerger le phénomène des milices a été décisif dans la constitution d'une armée unifiée.

11. Contrairement à ce que pense B. Ghalioun ("La stratégie à l'heure du nouvel ordre mondial. Vers une nouvelle règle du jeu entre le Nord et le Sud", Peuples méditerranéens, n58-59, 1992, pp. 247-258), on peut imaginer que l'ébranlement du statu quo n'est pas dans la logique de l'Etat-Nation, mais au contraire, en traduit la crise.

12. A la fois en Irak et en Turquie, certains éléments kurdes ont été organisés par l'Etat, qui les a armés et salariés. Ils ont été utilisés contre les guérilleros kurdes.

13. Il suffit de penser aux deux conférences internationales, de Paris, en 1989, et de Washington, en 1991, organisées par l'Institut Kurde de Paris.

14. Il est évident que l'opinion publique occidentale peut admettre la mobilisation humanitaire, voire la guerre, à condition que cela provoque pas des pertes en vies humaines. Cf. C. Le Borgne, "Zéro-Mort", in Le monde des Débats, Février 1992, p. 5.

15. Cf. Augstein (R.), "Pourquoi se mêler des luttes tribales", traduit de Der Spiegel in Le Courrier International, $\mathrm{n}^{\circ} 129,1993, \mathrm{p} .9$.

16. Répondant à un Kurde réclamant la proclamation de l'indépendance, J. Talabani expliquait : "...If right now we would declare independance, even without any military attack against us, Turkey and Iran would close their borders. Within a one week we will die. ... what you said is not a possibility for a responsbile leadership. It can be a poem, a 
good and nice poem by a poet, but not a plan by a realistic leadership", in F. Hussein, M. Leezenberg, P. Muller, The Reconstruction and Economic Development of Iraqi Kurdistan. Challenges and Perspectives, Amsterdam, Netherlands Kurdistan Society, 1993, p. 102.

17. Cf. pour cette notion, G. Nicolas, "De l'usage des victimes dans les stratégies politiques contemporaines", Culture et Conflit, nº, 1992-1993, pp. 129-163.

18. Cf. notre article, "L'Irak et ses communautés", Les Cahiers d'Orient, $n^{\circ} 29,1993$, pp. 111-119.

19. Notamment en Turquie où Jalal Talabani a été à l'origine du cessez-le-feu unilatéral décrété par le P.K.K.

20. Cf. l'article de F. Georgeon, "De Moussoul à Kirkouk. La Turquie et la question du Kurdistan irakien", Magheb Machrek, n¹32, 1991, pp.38-49.

21. Cf. notre article, "La régionalisation du problème kurde", in E. Picard (éd.) Nouvelle dynamique au Moyen-Orient. Turquie et Monde Arabe dans les années 80., Paris, l'Harmattan, 1993, pp. 166-181.

22. A titre d'exemple : « La fin de Saddam est arrivée. Après la guerre, la carte changera dans cette région. De nombreuses conférences auront lieu pour créer une nouvelle formation. Après la guerre nous ne nous assiérons pas à un banquet, mais à la table de négociations » in Cumhuriyet. 31 janvier 1991.

23. Cf. Chipaux (F.), "Le Kurdistan irakien sous perfusion", Le Monde, 18 juin 1993.

24. Smouts (M.C), "L'ordre neuf du président Bush", in Esprit, Les Cahiers de l'Orient, juin 1991, pp.181-188.

25. En 1992, l'Allemagne a suspendu ses livraisons d'armes à la Turquie à cause de sa politique kurde, alors qu'Ankara a trouvé plutôt un soutien de la part des autres capitales occidentales.

\section{RÉSUMÉS}

L'opération «humanitaire» «Provide comfort», qui comme le rappelle à juste titre l'auteur constitue juridiquement une intervention dans les affaires "intérieures" d'un Etat-membre de l'ONU, a largement dépassé les intentions initiales des Occidentaux et également celles des Kurdes, puisqu'elle a abouti à la création d'une entité territoriale kurde. Si l'opération n'a toutefois pas conduit à la défaite finale, de Saddam Hussein, de même à la "partition de l'Irak", la contestation kurde du pouvoir irakien sur le kurdistan est un état de fait, bien réel, et la souverainté territoriale irakienne est écornée. Le cas kurde malgré son évident aspect géopolitique présente malgré tout des points communs avec les autres opérations humanitaires puisqu'elle procède de la volonté de suspendre les conflits : véritable politique d'ajournement, ou de suspension de la guerre, évitant les génocides. Il oscille entre géopolitique et humanitaire ! L'analyse des relations entre les acteurs régionaux (Turquie, Iran, Irak, Etats-Unis, etc.), partie prenante et/ou confrontés au problème kurde, permet de mieux comprendre l'intersection de la logique humanitaire au sein d'un contexte hautement géopolitique sa logique et peut-être les raisons de son "succès". Elle s'inscrit en outre, et c'est une certitude, dans un processus de crise de l'Etat Nation et d'amplification des conflits ethniques d'une manière générale. 
INDEX

Index géographique : Irak, Kurdistan

Mots-clés : humanitaire, intervention militaire

Index chronologique : 1991 